\title{
BMJ Open Are low levels of serum bicarbonate associated with risk of progressing to impaired fasting glucose/diabetes? A single-centre prospective cohort study in Beijing, China
}

\author{
Sen Li, ${ }^{1,2}$ Ying-Ying Wang, ${ }^{1,2}$ Jing Cui, ${ }^{3}$ Dong-Ning Chen, ${ }^{3} \mathrm{Yu} \mathrm{Li}^{3}{ }^{3}$ Zhong Xin, ${ }^{1,2}$ \\ Rong-Rong Xie, ${ }^{1,2}$ Xi Cao, ${ }^{1,2}$ Jing Lu, ${ }^{1,2}$ Fang-Yuan Yang, ${ }^{2}$ Jin-Kui Yang ${ }^{1,2}$
}

To cite: Li S, Wang Y-Y, Cui J, et al. Are low levels of serum bicarbonate associated with risk of progressing to impaired fasting glucose/ diabetes? A single-centre prospective cohort study in Beijing, China. BMJ Open 2018;8:e019145. doi:10.1136/ bmjopen-2017-019145

- Prepublication history and additional material for this paper are available online. To view these files, please visit the journal online (http://dx.doi. org/10.1136/bmjopen-2017019145).

Received 14 August 2017 Revised 29 May 2018 Accepted 7 June 2018

\section{Check for updates}

C C Author(s) (or their employer(s)) 2018. Re-use permitted under CC BY-NC. No commercial re-use. See rights and permissions. Published by BMJ.

${ }^{1}$ Department of Endocrinology, Beijing Tongren Hospital, Capital Medical University, Beijing, China

${ }^{2}$ Beijing Key Laboratory of Diabetes Research and Care, Beijing, China

${ }^{3}$ Health Examination Center, Beijing Tongren Hospital, Capital Medical University, Beijing, China

Correspondence to Dr Jin-Kui Yang; jkyang@ccmu.edu.cn

\section{ABSTRACT}

Aims Bicarbonate is involved in many human essential metabolic processes, but little is known about the association between serum bicarbonate and glucose metabolism. This study aims to investigate the association between serum bicarbonate and the risk of progressing to impaired fasting glucose (IFG)/diabetes mellitus (DM).

Setting The data were obtained from a large-scale prospective cohort study in a single health centre in Beijing.

Participants A total of 5318 participants aged 18-70 years who underwent health examinations annually with baseline fasting plasma glucose (FPG) ranging from 3.9 to $5.5 \mathrm{mmol} / \mathrm{L}$, without a history of either diabetes or concomitant chronic diseases, were enrolled in this 6-year observational study.

Primary outcome measures A logistic regression analysis was used to calculate ORs for progressing to IFG/DM by the category of baseline serum bicarbonate. In addition, an analysis of the receiver operating characteristic (ROC) curve for predicting IFG was performed.

Results Of the 5318 participants, 210 developed IFG after a median 2.2 years of follow-up. After adjusting for sex, age, FPG, body mass index, systolic blood pressure, serum creatinine, serum alanine aminotransferase and lowdensity lipoprotein cholesterol at baseline, the participants in the first (OR 4.18, 95\% Cl 2.42 to $7.21 ; \mathrm{p}<0.001)$, second (OR 3.02, $95 \% \mathrm{Cl} 1.71$ to $5.33 ; \mathrm{p}<0.001)$ and third (OR 2.12, 95\% Cl 1.15 to $3.89 ; p=0.015$ ) quartiles of serum bicarbonate had higher odds for progressing to IFG/ DM compared with those in the highest quartile. The area under the ROC curve for predicting IFG/DM was 0.69 (95\% $\mathrm{Cl} 0.65$ to $0.72 ; p<0.001$ ).

Conclusions Lower serum bicarbonate is associated with higher risk of the development of IFG/DM.

\section{INTRODUCTION}

The increasing prevalence of pre-diabetes and diabetes is a prominent threat to public health. ${ }^{1}$ People with impaired fasting glucose (IFG) are asymptomatic and are at high risk

\section{Strengths and limitations of this study}

- This is the first study to evaluate the relationship between serum bicarbonate and the risk of progressing to impaired fasting glucose (IFG)/diabetes mellitus (DM).

- A large number of participants who completed a medical history questionnaire were involved in this study.

- There are insufficient data to determine the mechanism of the association between low bicarbonate and progression to IFG/DM.

- This work needs validation in another independent cohort to prove reproducibility.

of developing diabetes ${ }^{2}$ and cardiovascular disease. ${ }^{3}$ Without a change in lifestyle or appropriate treatment, approximately $9 \%$ of patients with IFG will develop type 2 diabetes mellitus (T2DM) within 3 years. ${ }^{4}$

IFG is characterised by a reduction in earlyphase insulin secretion, a severe hepatic insulin resistance and normal or near-normal muscle insulin resistance. ${ }^{56}$ An in vitro study showed that isolated rat islets incubated in the absence of bicarbonate had a completely inhibited first phase of glucose-stimulated insulin secretion (GSIS), and the second phase was reduced by approximately $65 \%{ }^{7}$ Meanwhile, a previous cohort study demonstrated that high-serum bicarbonate is associated with low risk of progression to T2DM in women. ${ }^{8}$ Bicarbonate-rich isotonic fluid is secreted by the exocrine pancreas and is involved in many essential human metabolic processes and maintains the acid-base balance. ${ }^{9}$ Exocrine pancreatic insufficiency in humans may be associated with insulin deficiency and diabetes mellitus (DM).${ }^{1011}$ In diabetic rats, impaired GSIS is coupled with 
exocrine pancreatic lesions, suggesting that changes in the islet microenvironment lead to the in vivo malfunction of $\beta$ cells. ${ }^{12}$ In addition, low bicarbonate is independently associated with insulin resistance. ${ }^{13}$ However, the effect of bicarbonate on IFG remains relatively undefined.

The current large-scale cohort study was therefore designed to assess the association between bicarbonate and the risk of progression to IFG/DM.

\section{METHODS}

\section{Participants}

The present study was performed in the physical examination centre of Beijing Tongren Hospital, Capital Medical University, Beijing, China. From January 2006 to December 2006, a total of 12001 participants accepted physical examination. After screening the patients for eligibility, 8107 participants aged $18-70$ years old with fasting plasma glucose (FPG) ranging from 3.9 to $5.5 \mathrm{mmol} / \mathrm{L}$ were chosen to take part in the study. The major exclusion criteria included prior diagnosis of diabetes, thyroid-related disease, renal disease, fatty liver disease, coronary heart disease, New York Heart Association Class III/IV heart failure or cancer. Diet, alcohol consumption and smoking status were self-reported and were assessed by use of semiquantitative questionnaires. Patients taking medications that could affect the results, such as diuretics or antacid medications at the baseline or during the observation, were excluded as well. From January 2007, the participants received telephone calls every 3 months, and were required report on self-monitored plasma glucose, blood pressure, exercise frequency and medication use. Each participant came to the examination centre annually for physical and laboratory examinations. Hypertension was defined as either systolic blood pressure (SBP) $\geq 140 \mathrm{~mm} \mathrm{Hg}$, diastolic blood pressure $(\mathrm{DBP}) \geq 90 \mathrm{~mm} \mathrm{Hg}$ or use of antihypertensive medications.

The follow-up of the last participant was completed in December 2012. From 2007 to 2012, the number of participants at each visit was 7742 (95.5\%), 7272 (89.7\%), 6656 (82.1\%), $6081(75.0 \%), 5627(69.4 \%)$ and $5118(63.1 \%)$ (not including those who had reached IFG/DM). After 6 years of follow-up, 5318 participants had completed all the visits, 1622 withdrew from the follow-up, 1139 failed to contact and 28 died.

\section{Definition of progressing to IFG/DM}

We used a modified version of the diagnostic criteria recommended by the American Diabetes Association (ADA) in 1997 as our definition of IFG. The ADA defined IFG as FPG $\geq 6.1 \mathrm{mmol} / \mathrm{L}(\geq 110 \mathrm{mg} / \mathrm{dL})$ and $<7.0 \mathrm{mmol} / \mathrm{L}$ $(<126 \mathrm{mg} / \mathrm{dL})$ and DM as FPG $\geq 7.0 \mathrm{mmol} / \mathrm{L}(\geq 126 \mathrm{mg} /$ dL) ${ }^{14}$ In this study, the participants with an FPG from 3.9 to $5.5 \mathrm{mmol} / \mathrm{L}$ at baseline and with an FPG $\geq 6.1 \mathrm{mmol} / \mathrm{L}$ (including $\geq 7.0 \mathrm{mmol} / \mathrm{L}$ ) after follow-up were defined as 'progressing to IFG/DM'.

\section{Laboratory procedures}

All the participants who enrolled in our study received physical examinations, and their blood samples were collected. Weight and height were measured during the physical examination (MW-900A, Lejia, Hebei, China) and were used to calculate body mass index (BMI) (kg/ $\mathrm{m}^{2}$ ). Blood pressure was measured with the person in a seated position after a $5 \mathrm{~min}$ rest with an electronic blood pressure monitor (TM-2656VP, Aieande, Japan). Morning blood was sampled to make sure the patients were fasting for more than 6 hours. Serum bicarbonate, which was measured using an indirect ion-selective electrode method, and other biochemical parameters, including FPG, total cholesterol (TC), triglycerides (TG), low-density lipoprotein cholesterol (LDL-C), high-density lipoprotein cholesterol, serum creatinine (Cr), alanine aminotransferase (ALT) and aspartate aminotransferase (AST), were all measured by a UniCel DxC 800 automatic biochemistry analyser (Beckman Coulter, CA, USA).

\section{Statistical analyses}

The results for continuous variables in the cohort study were given as mean $\pm \mathrm{SD}$, and the proportions were given as percentages. We divided the data at the median and into quartiles according to the serum bicarbonate levels at baseline..$^{15}$ The baseline characteristics of each group were compared by a $\chi^{2}$ test or a one-way analysis of variance test. Three logistic regression models were used to calculate the ORs of progressing to IFG/DM. We considered those variables possibly related to blood glucose and bicarbonate levels as potential confounders for adjustment. First, we performed logistic regression analyses between bicarbonate and each confounder separately to obtain crude OR values, the factors whose $\mathrm{p}<0.2$ in this step were selected to make further adjustments. Model 1 was unadjusted. Model 2 was adjusted for age, FPG and sex. Model 3 was adjusted for age, FPG, sex, BMI, SBP, serum Cr, LDL-C and ALT. A subgroup analysis was performed in the logistic regression model 3 between individuals with serum bicarbonate below the median level and those with serum bicarbonate above the median level. The subgroups were categorised by age, sex, BMI, SBP and LDL-C. The receiver operating characteristic (ROC) curve of serum bicarbonate was conducted for predicting IFG/DM. The optimum cut-off point was defined as the point that had the maximum sensitivity plus specificity. In addition, we calculated the prevalence of IFG/DM by deciles of the distribution of serum bicarbonate values. Statistical analyses were performed using SPSS V.19.0 statistical software (SPSS). $\mathrm{P}<0.05$ was considered significant.

\section{Patient and public involvement}

The development of the research questions and outcome measures was not informed by patients' priorities, experience or preferences. Participants will be sent a short message or an email of the brief findings when the main article is published. 
Table 1 Baseline characteristics of participants by quartile of serum bicarbonate

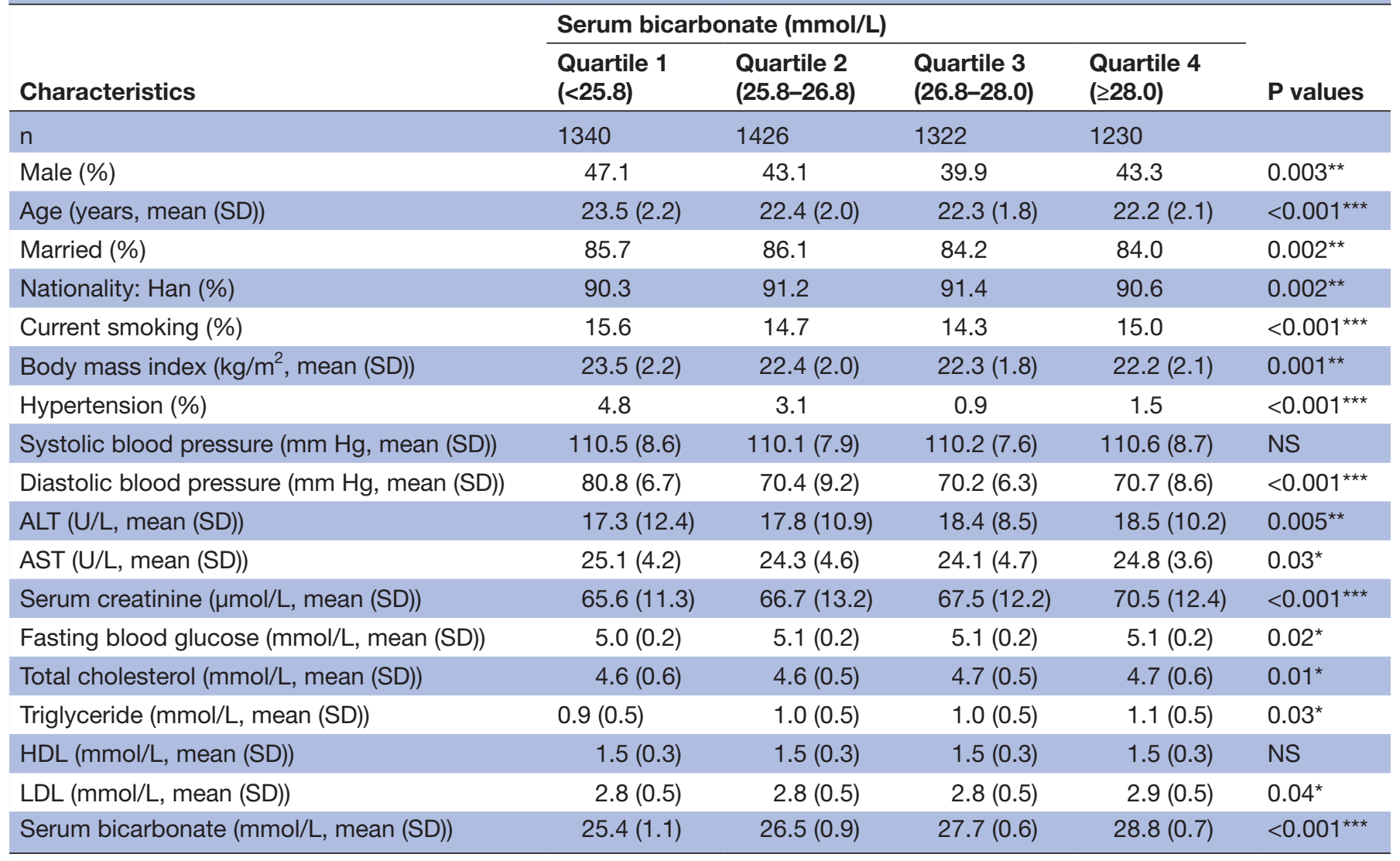

Data are mean (SD) unless otherwise indicated.

$P$ values are from one-way analysis of variance (ANOVA) for those reported as means and $\chi^{2}$ test for those reported as percentages. $P$ values are divided into four categories and footnoted:

${ }^{*} \mathrm{P}<0.05 ;{ }^{* *} \mathrm{P}<0.01$; ${ }^{* *} \mathrm{P}<0.001$

ALT, alanine aminotransferase; AST, aspartate aminotransferase; HDL, high-density lipoprotein; LDL, low-density lipoprotein; NS, no significance.

\section{RESULTS}

\section{Baseline characteristics}

A total of 5318 participants (male 2307, female 3011) completed the follow-up and were enrolled in the cohort study; the baseline characteristics were categorised by quartile of serum bicarbonate levels and are shown in table 1. The bicarbonate levels analysed ranged from 20.1 to $31.0 \mathrm{mmol} / \mathrm{L}$, with mean $\pm \mathrm{SD}(26.7 \pm 2.2) \mathrm{mmol} / \mathrm{L}$ and median (IQR) $26.8(25.3-28.4) \mathrm{mmol} / \mathrm{L}$; the normal reference values in our lab for serum bicarbonate are $24.2-30.7 \mathrm{mmol} / \mathrm{L}$. The participants with the lowest levels of serum bicarbonate were more likely to be male and older in age; these participants also had higher BMI, SBP, DBP, ALT and AST and had a greater chance of a history of hypertension. The participants with the lowest levels of serum bicarbonate had lower levels of FPG, serum Cr, TC, TG and LDL-C. We compared the demographic and laboratory data between those who dropped out and those participants who continued in the study; there was no statistically significant difference.

\section{Follow-up analysis}

After a median 2.2 years of follow-up, 210 (male, 122; female, 88) participants developed IFG/DM. The number of IFG/DM events by groups (serum bicarbonate low to high) was 57, 55, 52 and 46 (table 2). In the unadjusted logistic regression model 1 , the participants in the first (OR $6.58,95 \%$ CI 3.87 to 11.20 ; $\mathrm{p}<0.001$ ), second (OR 2.99, $95 \%$ CI 1.70 to $5.25 ; \mathrm{p}<0.001)$ and third (OR $1.94,95 \%$ CI 1.06 to $3.55 ; \mathrm{p}=0.031$ ) quartiles of serum bicarbonate had higher odds of IFG/DM compared with those in the fourth quartile (table 2). The univariate logistic regression analysis to assess the association between serum bicarbonate and the risk of development of IFG/DM yielded an OR of 3.16 (95\% CI 2.28 to $4.37 ; \mathrm{p}<0.001$ ) when comparing the participants with the serum bicarbonate below the median level versus those with the serum bicarbonate above the median level (online supplementary table S1).

In models 2 and 3 , after adjusting for different variables, the ORs were attenuated but remained significant. The participants with serum bicarbonate below the median level had higher odds of incident IFG/DM of 2.34 (95\% CI 1.67 to $3.28 ; p<0.001)$ compared with those above the median with adjustment in model 3 (online supplementary table S1).

The area under the ROC curve (AUC) of serum bicarbonate for predicting IFG/DM was 0.69 (95\% CI 0.65 to 
Table 2 ORs of impaired fasting glucose in groups by quartile of baseline serum bicarbonate

\begin{tabular}{|c|c|c|c|c|}
\hline Groups and models & Quartile of serum bi & nate (mmol/L); OR (95 & $\mathrm{Cl}) \dagger$ & \\
\hline All & Quartile 1 (<25.8) & Quartile 2 (25.8-26.8) & Quartile 3 (26.8-28.0) & Quartile 4 (>28.0) \\
\hline IFG events & 57 & 55 & 52 & 46 \\
\hline Model 2 & 4.52 (2.62 to 7.77$)$ & 2.96 (1.68 to 5.20$)$ & $2.17(1.18 \text { to } 3.97)^{\star \star}$ & Reference \\
\hline Model 3 & 4.18 (2.42 to 7.21$)$ & 3.02 (1.71 to 5.33$)$ & $2.12(1.15 \text { to } 3.89)^{\star \star \star}$ & Reference \\
\hline
\end{tabular}

Model 1: unadjusted.

Model 2: adjusted for age, sex and fasting blood glucose (FBG).

Model 3: model 2+adjusted for body mass index, systolic blood pressure, serum creatinine, low-density lipoprotein (LDL)

and alanine aminotransferase (ALT).

${ }^{*} \mathrm{P}=0.031 ;{ }^{\star \star} \mathrm{P}=0.012 ;{ }^{\star \star \star} \mathrm{P}=0.015$.

$P$ values for all other models were $<0.001$.

†Unless otherwise stated.

IFG, impaired fasting glucose.

0.72). The analysis of the ROC curve yielded a sensitivity of $61.9 \%$, specificity of $68.1 \%$, positive predictive value of $7.4 \%$ and a negative predictive value (NPV) of $97.8 \%$. The optimum cut-off value (figure 1) to predict IFG was 26.1 (25.30-28.42) $\mathrm{mmol} / \mathrm{L}$ of serum bicarbonate. Figure 2 shows the prevalence of IFG/DM by deciles of the distribution of serum bicarbonate values. As the concentration of serum bicarbonate decreased, the percentage of participants progressing to IFG/DM increased. The participants in the first three deciles (with serum bicarbonate $<26.0 \mathrm{mmol} / \mathrm{L}$ ) had a higher prevalence of IFG than the participants in the 4th to 10th deciles (with serum bicarbonate $>26.0 \mathrm{mmol} / \mathrm{L}$ ).

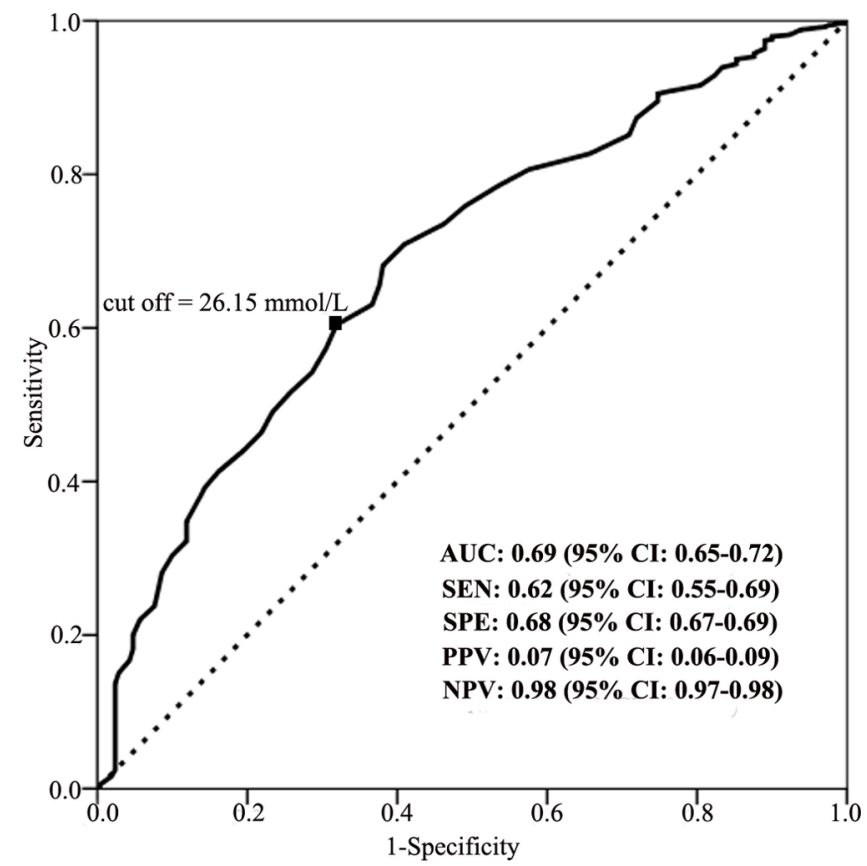

Figure 1 Receiver operating characteristic (ROC) curve of serum bicarbonate for predicting impaired fasting glucose (IFG). The optimal cut-off point for serum bicarbonate was $26.15 \mathrm{mmol} / \mathrm{L}$. AUC, area under the ROC curve; NPV, negative predictive value; PPV, positive predictive value; SEN, sensitivity; SPE, specificity.
Logistic regressions were conducted by the cut-off value of the ROC curve. The participants with serum bicarbonate below the cut-off points had ORs of $3.47(95 \%$ CI 2.61 to $4.61 ; \mathrm{p}<0.001$ ) for model 1, 2.40 (95\% CI 1.77 to 3.26; $\mathrm{p}<0.001$ ) for model 2 and 2.32 (95\% CI 1.71 to $3.16 ; \mathrm{p}<0.001$ ) for model 3 (online supplementary table S2). In model 3, the logistic regression results matched closely with the results obtained by the 'high' and 'low' categorisations of the median serum bicarbonate level (online supplementary table S3).

\section{DISCUSSION}

In this study, we observed that low levels of serum bicarbonate increased the prevalence of IFG/DM independent of risk factors, including age, sex, baseline FPG, BMI, SBP,

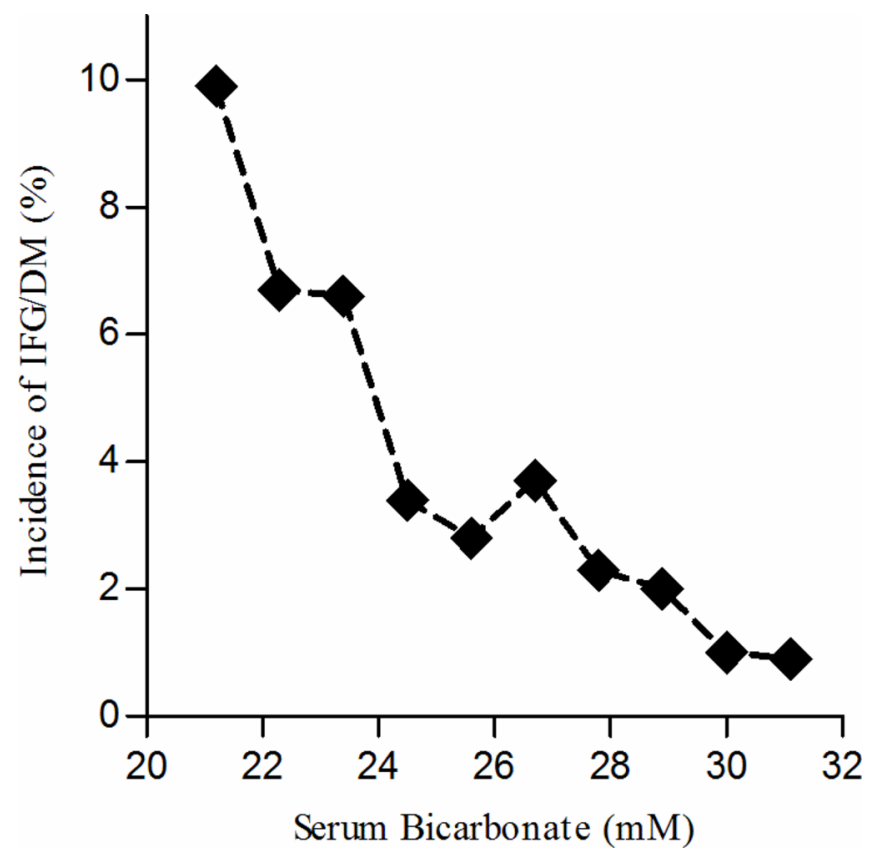

Figure 2 Prevalence of impaired fasting glucose (IFG) by deciles of the baseline serum bicarbonate. DM, diabetes mellitus. 
serum Cr, LDL-C and ALT. In addition, the results of the AUC suggested that serum bicarbonate concentration had a moderate but significant predicting ability on the development of IFG/DM.

A prospective, nested case-control study among women, $98 \%$ were white, found an association between higher plasma bicarbonate concentration and lower odds of incident T2DM. ${ }^{8}$ Although the authors did not address the specific mechanism, they suggested this association may be explained by metabolic acidosis promoting insulin resistance. Various theories have been proposed to mediate the influence of dietary acid load and insulin resistance, including decreased insulin-stimulated glucose uptake in muscle, disrupted binding of insulin to the insulin receptor and inhibited insulin signalling pathway. ${ }^{16}$ This work is consistent with previous observations; Redondo et al demonstrated that insulin deficiency is a major contributor to diabetic ketoacidosis (DKA) but, conversely, the metabolic effects of ketoacidosis may temporarily decrease further the ability to secrete insulin. ${ }^{17}$ A probable relationship links the low-pH environment and impaired insulin secretion, but the precise mechanism needs more exploration. As mentioned above, IFG is characterised by a reduction in early-phase insulin secretion and severe hepatic insulin resistance. ${ }^{56}$ Thus far, there has been little research elucidating the effect of bicarbonate on insulin secretion.

Ion channels play an important role in the regulation of pancreatic $\beta$-cell electrical activities and, thus, in GSIS. ${ }^{18}$ At high-glucose concentrations, the closure of the ATP-sensitive potassium channels ( $\mathrm{K}_{\mathrm{ATP}}$ channels) leads to depolarisation of $\beta$ cells and the activation of $\mathrm{Ca}^{2+}$ influx. The increase in intracellular $\mathrm{Ca}^{2+}$ facilitates insulin granule exocytosis and promotes insulin secretion. ${ }^{18} 19$ Recently, cystic fibrosis transmembrane conductance regulator (CFTR) was proved to modulate the glucose-induced electrical activities and insulin secretion in pancreatic $\beta$ cells. ${ }^{20}$ CFTR is a cAMP-regulated chloride channel that has been proved to contribute to the transport of bicarbonate. ${ }^{21} 22$ The expression of CFTR in pancreatic $\beta$ cells may be influenced by different concentrations of extracellular bicarbonate, which leads to a different level of insulin secretion. The exocrine and endocrine parts of the pancreas interact with each other, and the exocrine insufficiency leads to the dysfunction of endocrine islets. Recently, Fortunato et al demonstrated autophagy and necroptosis signalling were also involved in exocrine insufficiency, suggesting that new signalling pathway might participate in the regulation of islet glucose metabolism. ${ }^{23}$ Nevertheless, the precise mechanism underlying the association between bicarbonate and insulin secretion awaits further investigation.

We have noticed the unexpected relationship between serum bicarbonate and FPG, serum Cr, TC, TG and LDL at baseline. The results in table 1 were obtained in 5318 participants at baseline, where there was a bias for sex distribution (male 44\%). A total of 8107 participants were admitted, but more than 2000 withdrew or were lost to follow-up. We made another statistical analysis of these characteristics for all the 8107 participants (male 48\%), and we found no significant difference among the four groups. These biochemical parameters can be influenced by many other factors, such as diet or activity. The association with bicarbonate needs to be validated by another logistic regression statistical analysis.

A U-shaped association between serum bicarbonate levels and mortality has been shown in various studies of chronic kidney disease (CKD). Dobre et al demonstrated that in patients with CKD, persistent serum bicarbonate $>26 \mathrm{mmol} / \mathrm{L}$ was associated with increased risk of heart failure events and mortality. However, in another study among National Health and Nutrition Examination Survey III participants, low-serum bicarbonate was not observed to be a strong predictor of mortality in people without CKD. The present non-CKD research is not contradicted with these two studies, but our data should be interpreted with caution.

The AUC of the ROC curve of serum bicarbonate was 0.69 , which indicates a poor discrimination for serum bicarbonate of predicting IFG/DM. However, the $97.8 \%$ NPV results demonstrated great predictive value for the absence of development of IFG/DM. Bicarbonate above a certain level is protective. The optimum cut-off value for predicting progression to $\mathrm{IFG} / \mathrm{DM}$ was $26.1 \mathrm{mmol} / \mathrm{L}$ for serum bicarbonate, which was very close to the median level. The mechanism of progressing to IFG/DM is complicated and should not be predicted by a single parameter. Low bicarbonate is a necessary but not sufficient cause of progression to IFG/DM.

However, the current study has several limitations. First, there is a lack of data to shed light on the mechanism of the association between low bicarbonate and progression (eg, no data on insulin resistance markers, insulin secretion or pancreatic exocrine insufficiency). Second, this is a single-centre study in an Asian population, which limits the generalisation of the conclusion. Third, we do not have data on arterial or venous $\mathrm{pH}$, which limits the ability to precisely characterise the acid status of the participants. Additionally, a single measurement of bicarbonate decreases the accuracy of the data, and whether dietary supplementation of alkali would increase the insulin secretion or reduce the risk of IFG/ DM is not discussed. Other limitations are the sizeable proportion of lost-to-follow-up participants; the adjustment for confounder variables influencing the glucose metabolism may have been incomplete, such as dietary variables, especially water/beverage drinking habits and the consumption of medications that may change the $\mathrm{pH}$. These findings need validation in an independent cohort to prove reproducibility.

In conclusion, our present research found an association between lower serum bicarbonate and higher risk of progressing to IFG/DM in Chinese people. The prevention of IFG/DM could benefit from controlling serum bicarbonate at a relatively higher level within the normal range in the population. Subsequent studies are required 
to explore the underlying mechanism in which the regulation of insulin resistance or insulin secretion could be involved.

Acknowledgements The authors thank all the people who participated in the study.

Contributors JKY designed the study, conducted the experiments and wrote the article. SL, YYW and JC conducted the experiments, performed the data analysis and wrote the article. DNC, YL and ZX conducted the experiments and performed the data analysis. RRX, XC, FYY and JL conducted the experiments. All the authors critically revised or drafted the manuscript for important intellectual content.

Funding This work was supported by the National Natural Science Foundation of China (8151101058; 81471014; 81270918), the Beijing Natural Science Foundation (7131005) and the Beijing Municipal Science and Technology Project (D12110000412001) to JKY.

Competing interests None declared.

Patient consent Obtained.

Ethics approval Medical Ethics Committee of Beijing Tongren Hospital, Capital Medical University.

Provenance and peer review Not commissioned; externally peer reviewed.

Data sharing statement All the available data can be obtained by contacting the corresponding author.

Open access This is an open access article distributed in accordance with the Creative Commons Attribution Non Commercial (CC BY-NC 4.0) license, which permits others to distribute, remix, adapt, build upon this work non-commercially, and license their derivative works on different terms, provided the original work is properly cited, appropriate credit is given, any changes made indicated, and the use is non-commercial. See: http://creativecommons.org/licenses/by-nc/4.0/.

\section{REFERENCES}

1. Barcelo A, Gregg EW, Gerzoff RB, et al. Prevalence of diabetes and intermediate hyperglycemia among adults from the first multinational study of noncommunicable diseases in six Central American countries: the Central America Diabetes Initiative (CAMDI). Diabetes Care 2012;35:738-40.

2. Nóvoa FJ, Boronat M, Saavedra P, et al. Differences in cardiovascular risk factors, insulin resistance, and insulin secretion in individuals with normal glucose tolerance and in subjects with impaired glucose regulation: the Telde Study. Diabetes Care 2005;28:2388-93.

3. Pankow JS, Kwan DK, Duncan BB, et al. Cardiometabolic risk in impaired fasting glucose and impaired glucose tolerance: the Atherosclerosis Risk in Communities Study. Diabetes Care 2007;30:325-31.

4. Nichols GA, Hillier TA, Brown JB. Progression from newly acquired impaired fasting glusose to type 2 diabetes. Diabetes Care 2007;30:228-33.
5. Abdul-Ghani MA, Jenkinson CP, Richardson DK, et al. Insulin secretion and action in subjects with impaired fasting glucose and impaired glucose tolerance: results from the Veterans Administration Genetic Epidemiology Study. Diabetes 2006;55:1430-5.

6. Weyer C, Bogardus C, Pratley RE. Metabolic characteristics of individuals with impaired fasting glucose and/or impaired glucose tolerance. Diabetes 1999;48:2197-203.

7. Henquin JC, Lambert AE. Extracellular bicarbonate ions and insulin secretion. Biochim Biophys Acta 1975;381:437-42.

8. Mandel El, Curhan GC, Hu FB, et al. Plasma bicarbonate and risk of type 2 diabetes mellitus. CMAJ 2012;184:E719-E725.

9. Futakuchi $\mathrm{S}$, Ishiguro $\mathrm{H}, \mathrm{Naruse} \mathrm{S}$, et al. High glucose inhibits $\mathrm{HCO} 3(-)$ and fluid secretion in rat pancreatic ducts. Pflugers Arch 2009;459:215-26.

10. Wooldridge JL, Szczesniak RD, Fenchel MC, et al. Insulin secretion abnormalities in exocrine pancreatic sufficient cystic fibrosis patients. $J$ Cyst Fibros 2015;14:792-7.

11. Hardt PD, Brendel MD, Kloer HU, et al. Is pancreatic diabetes (type $3 c$ diabetes) underdiagnosed and misdiagnosed? Diabetes Care 2008;31(Suppl 2):S165-S169.

12. Weksler-Zangen S, Raz I, Lenzen S, et al. Impaired glucosestimulated insulin secretion is coupled with exocrine pancreatic lesions in the Cohen diabetic rat. Diabetes 2008;57:279-87.

13. Farwell WR, Taylor EN. Serum bicarbonate, anion gap and insulin resistance in the National Health and Nutrition Examination Survey. Diabet Med 2008;25:798-804.

14. Report of the expert committee on the diagnosis and classification of diabetes mellitus. Diabetes Care 1997;20:1183-97.

15. Paul Chubb SA, Davis WA, Peters KE, et al. Serum bicarbonate concentration and the risk of cardiovascular disease and death in type 2 diabetes: the Fremantle Diabetes Study. Cardiovasc Diabetol 2016;15:143

16. Gall WE, Beebe K, Lawton KA, et al. alpha-hydroxybutyrate is an early biomarker of insulin resistance and glucose intolerance in a nondiabetic population. PLoS One 2010;5:e10883.

17. Redondo MJ, Rodriguez LM, Escalante M, et al. Beta cell function and $\mathrm{BMI}$ in ethnically diverse children with newly diagnosed autoimmune type 1 diabetes. Pediatr Diabetes 2012;13:564-71.

18. Rorsman P, Braun M. Regulation of insulin secretion in human pancreatic islets. Annu Rev Physiol 2013;75:155-79.

19. Fridlyand LE, Jacobson DA, Philipson LH. Ion channels and regulation of insulin secretion in human $\beta$-cells. Islets 2013;5:1-15.

20. Guo JH, Chen H, Ruan YC, et al. Glucose-induced electrical activities and insulin secretion in pancreatic islet $\beta$-cells are modulated by CFTR. Nat Commun 2014;5:4420.

21. Choi JY, Muallem D, Kiselyov K, et al. Aberrant CFTR-dependent HCO3- transport in mutations associated with cystic fibrosis. Nature 2001;410:94-7.

22. Shah VS, Ernst S, Tang XX, et al. Relationships among CFTR expression, $\mathrm{HCO} 3-$ secretion, and host defense may inform geneand cell-based cystic fibrosis therapies. Proc Natl Acad Sci U S A 2016;113:5382-7.

23. Zhou X, Xie L, Xia L, et al. RIP3 attenuates the pancreatic damage induced by deletion of ATG7. Cell Death Dis 2017;8:e2918. 